\title{
VERIFICATION OF AMBA AXI BUS PROTOCOL IMPLEMENTING INCR AND WRAP BURST USING SYSTEM VERILOG
}

\author{
Harsha Garua ${ }^{1}$, Keshav Sharma ${ }^{2}$, Chusen Duari ${ }^{3}$ \\ ${ }^{1}$ Manipal University Jaipur \\ ${ }^{2}$ Manipal University Jaipur \\ ${ }^{3}$ Assistant Professor, Manipal University Jaipur
}

\begin{abstract}
This paper describes the development of verification environment for AMBA AXI (Advanced Extensible Interface) protocol using System Verilog. AXI supports high performance, high-frequency system designs. It is an On-Chip communication protocol. It is suitable for high-bandwidth and high frequency designs with minimal delays. It provides flexibility in the implementation of interconnect architectures and avoid use of complex bridges. It is backward-compatible with existing AHB and APB interfaces. The key features of the AXI protocol are that it consists of separate address, control and data phases. It support unaligned data transfers using byte strobes. It requires only start address to be issued in a burst-based transaction. It has separate read and write data channels that provide low-cost Direct Memory Access (DMA). This paper is aimed at the verification of various burst type transaction (INCR and WRAP) of the AXI bus protocol and the Verification Environment is built using System Verilog coding[1].
\end{abstract}

Keywords: AMBA AXI, INCR, Wrap Burst, System Verilog

\section{INTRODUCTION}

AMBA stands for Advanced Microcontroller Bus Architecture (AMBA).It is an open-standard, on-chip interconnect specification for the connection and management of intellectual property (IP) blocks in systemon-a-chip (SoC) designs. It supports development of designs with large numbers of controllers and peripherals/IP blocks. Since its inception, the scope of AMBA has, despite its name, gone far beyond micro controller devices.

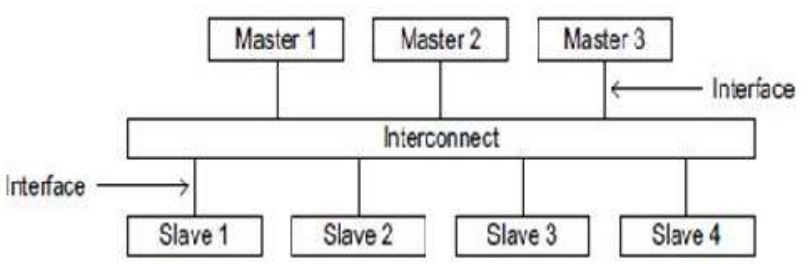

Figure 1: AXI Topology

The AXI protocol support burst-based transaction which signifies transfer of multiple data grouped in packets. It consists of independent transaction channels: Read Address Channel, Read Data Channel, Write Address Channel, Write Data Channel and the Write Response Channel. An address channel carries control information that describes the size of bursts, number of bursts and length of the data to be transferred. The data is transferred between master and slave using either:

- Write Data Channel: in write transaction data is transferred from the master to the slave using this channel.

- Read Data Channel: in read transaction data transferred from the slave to the master using this channel.
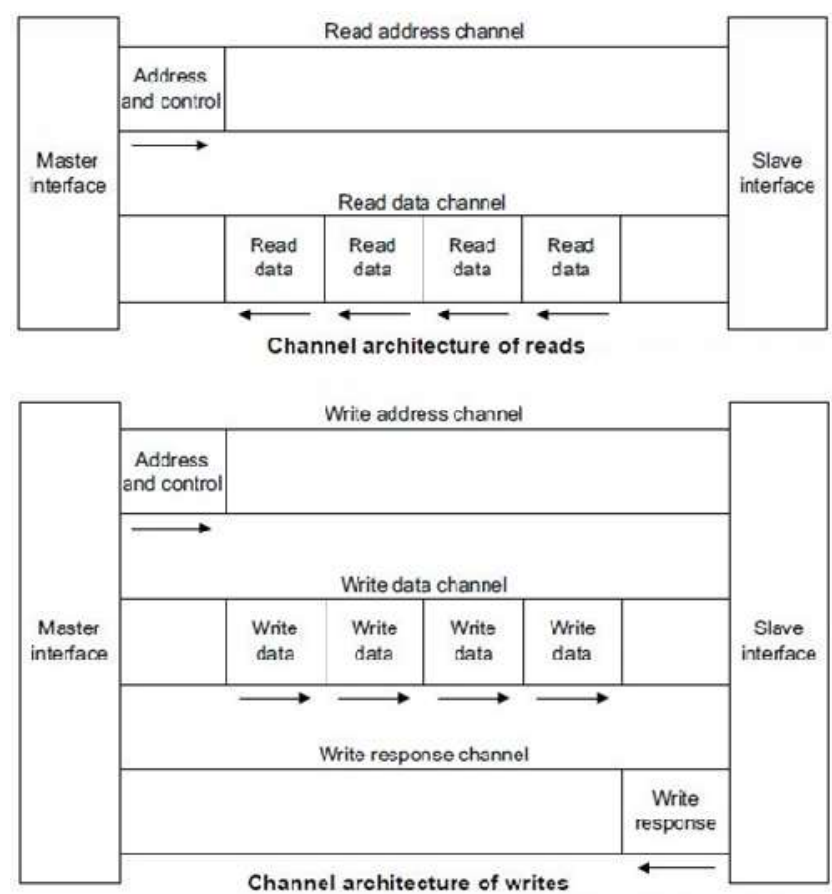

Figure 2: AXI Architecture

FIXED burst:

In a fixed burst-type, single address is used for storing data that is frequently used by the slave similar to a FIFO for every transfer in the burst. This type of burst enables repeated accesses to the same location.

INCR burst:

In an incrementing burst, the address is incremented for each transfer in the burst from the previous address. The size of burst determines the increment value for the address. 
Case I:

\begin{tabular}{cccrl} 
Address & \multicolumn{2}{c}{ ADR AxBURST } & AxSIZE & Data \\
0x9 & 0x9 & INCR & 8-bit & 1 data transfer \\
0xA & 0xA & INCR & 8-bit & 1 data transfer \\
0xB & 0xB & INCR & 8-bit & 1 data transfer \\
0xC & 0xC & INCR & 8-bit & 1 data transfer
\end{tabular}

Case II:

\begin{tabular}{ccccc} 
Address & ADDR AxBURST & AxSIZE & \multicolumn{2}{c}{ Data } \\
0x0 & 0x00 & INCR4 & 4-byte & 1 data transfer \\
0x4 & 0x04 & INCR4 & 4-byte & 1 data transfer \\
0x8 & 0x08 & INCR4 & 4-byte & 1 data transfer \\
0xC & 0x0C & INCR4 & 4-byte & 1 data transfer
\end{tabular}

Wrapping burst:

Similar to INCR a wrapping burst is also consists of incrementing the address for each transfer of the previous transfer address. In a wrapping burst when the boundary is reached the address wraps around to a lower address. The wrap boundary is determined by the size of each transfer in the burst multiplied by the total number of transfers in the burst. Two restrictions apply to wrapping bursts:

- WRAP can work only for the start address aligned to the size of the transfer.

- And the burst length must be $(2,4,8,16)$.

Case I: If we do 4 beat burst on 32 bit AXI with AWLEN=16 and starting address 0x00000004 address

\section{DUT}

The verification environment is organized in a hierarchical layered structure which helps to verification. The following subsections explain the functionality of four primary components in the verification environment.

\section{Virtual Interface}

Virtual interfaces provide a mechanism for separating abstract models and test programs from the actual signals that make up the design. It allows the same subprogram to operate on different portions of design, and to dynamically control the set of signals associated with the subprogram. Instead of referring to the actual set of signals directly, users are able to manipulate a set of virtual signals.

\section{Scoreboard}

The scoreboard collects address and control information on Write Address Channel and the data on Write Data Channel at the output of AXI slave and compares it with the address, data and control information at the output of DUV. It collects address and control information on Read Address Channel at the AXI slave input side and compares it with the address and control information at the output of the DUV. It collects the read response and data at the DUV side and compares it with that on the input side[4].

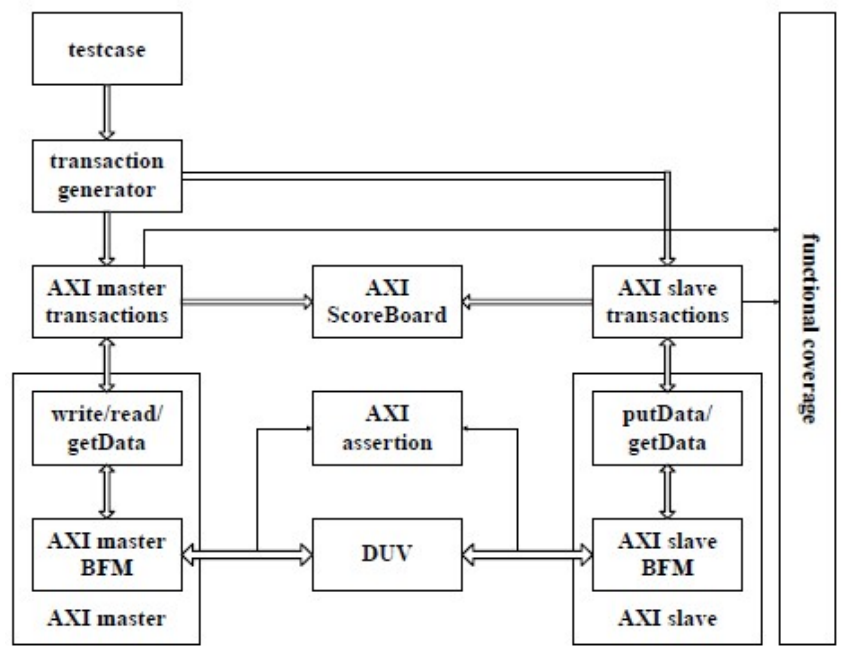

Figure 3: System Verilog Components

AXI stands for Advanced Extensible Interface. It is a part of the Advanced Microcontroller Bus Architecture (AMBA) developed by ARM (Advanced RISC Machines) company. It is an On-Chip communication protocol. The AMBA AXI protocol supports high-performance, high-frequency system designs. The key features of the AXI protocol are that it has separate address phase and data phases. It uses byte strobes to support unaligned data transfers. It utilizes burst-based transactions with only the start address issued. It has separate read and write data channels that provide support for Direct Memory Access (DMA) at a lower cost. It support for out-of-order transaction completion. It permits easy addition of register stages to provide timing closure.

Objective of paper:

- To study AMBA AXI Specification

- To implement INCR and WRAP burst type transaction in AXI design, design coding in system verilog

- To prepare testcases for various burst size and burst length.

- To achieve code and functional coverage of the verification environment developed

\section{AXI PROTOCOL SPECIFICATION}

A typical system-on-chip design consists of a number of master and slave devices connected together using the interconnecting protocol [2]. The AXI protocol provides a single interface definition, for the interfaces:

- Between a master and the interconnect

- Between a slave and the interconnect

- Between a master and a slave.

The key features of the AXI protocol are:

- Consists of separate address phase and data phase.

- support non-sequential data transfers using byte strobes

- data is transferred in form of packets i.e. bursts with only start address issued

- separate channels for read and write transactions to enable low-cost Direct Memory Access (DMA) 
- issue multiple outstanding addresses

- support out-of-order transactions

- supports addition of new register stages to provide timing closure.

- allows address information to be issued before the actual data transfer.

\section{Burst Operation in AXI}

The AXI protocol support is burst-based transaction. Every transaction consists of address and control information issued on the address channel which describes various parameters of the data to be transferred. A write data channel is used to transfer data between master and slave. The AXI protocol consists of an additional write response channel in which slave sends response to the master as an acknowledgement for the write transaction [5]. The AXI protocol enables:

- address information to be issued ahead of the actual data transfer.

- support for multiple outstanding transactions.

- support for out-of-order completion of transactions.

The AXI protocol defines three transaction bursts types described in:

- Fixed burst

- Incrementing burst

- Wrapping burst

Data Address

First: $\quad$ 0x00000004 0x00000004

Second: 0x00000008 0x00000008

Third: 0x0000000C 0x0000000C

Fourth 0x00000010 0x00000000 (Wrapped)

The WRAP operation address is wrapped to transfer size.

Case II: If we do 4 beat burst on 16 bit AXI with

AWLEN=4 and starting address 0x00000004 address

$$
\text { Data Address }
$$

First: $\quad$ 0x00000002 0x00000004

Second: 0x00000004 0x00000006

Third: 0x00000006 0x00000008

Fourth 0x00000008 0x00000002 (Wrapped)

The WRAP operation address is wrapped to transfer size.

\section{SYSTEM VERILOG BASED VERIFICATION}

The basic components of the verification environment are described below[3]:

1. Testbench

Testbench mimics the environment in which the design resides. It checks whether the real time logic implementation meets the design specification or not. This environment creates invalid and unexpected as well as valid and expected conditions to test the design under verification.

\section{Test Cases}

Test cases are written for different scenarios, which cover the functionality and corner cases. Basic read-write transactions and various burst types are verified. Parameterization will be randomized for different test cases and are written to check all the possible scenarios. These test cases are run in regression with multiple seeds.

\section{AXI Master}

The AXI master mainly consists of two classes, axi_m_driver and axi_m_env. The AXI master is implemented by the axi_m_driver class and the axi_m_env consists of the tasks from master transaction and generator class. The axi_m_driver class consists of a main task named run_m_driver() which drives packets from Interface to the DUV.

\section{AXI Slave}

The AXI slave consists of axi_s_driver class and axi_s_env class. The AXI slave is implemented by the axi_s_driver class and the axi_s_env runs the tasks of slave transaction and generator class. The axi_s_driver class has a run_s_driver() task which drives the generated signals from interface to the DUV in loops using mailbox element.

\section{AXI Assertion}

System Verilog assertion (SVA) has been used here to check the various properties of AXI interconnection for an expected behavior. In this paper, assertions are applied to detect a successful communication between master and slave.

\section{AXI Scoreboard}

The AXI scoreboard comprises of the comparison between the address and data send by an AXI master in a write/read transaction in a burst and the address and data in the slave. The scoreboard consists of the output values from the Golden Model and the DUV and sends it to the Checker block for analysis. Every transaction in AXI consists of a unique AWID or ARID that is issued according to the AXI protocol and the transactions can arrive in any order with different IDs. 


\section{WAVEFORM}

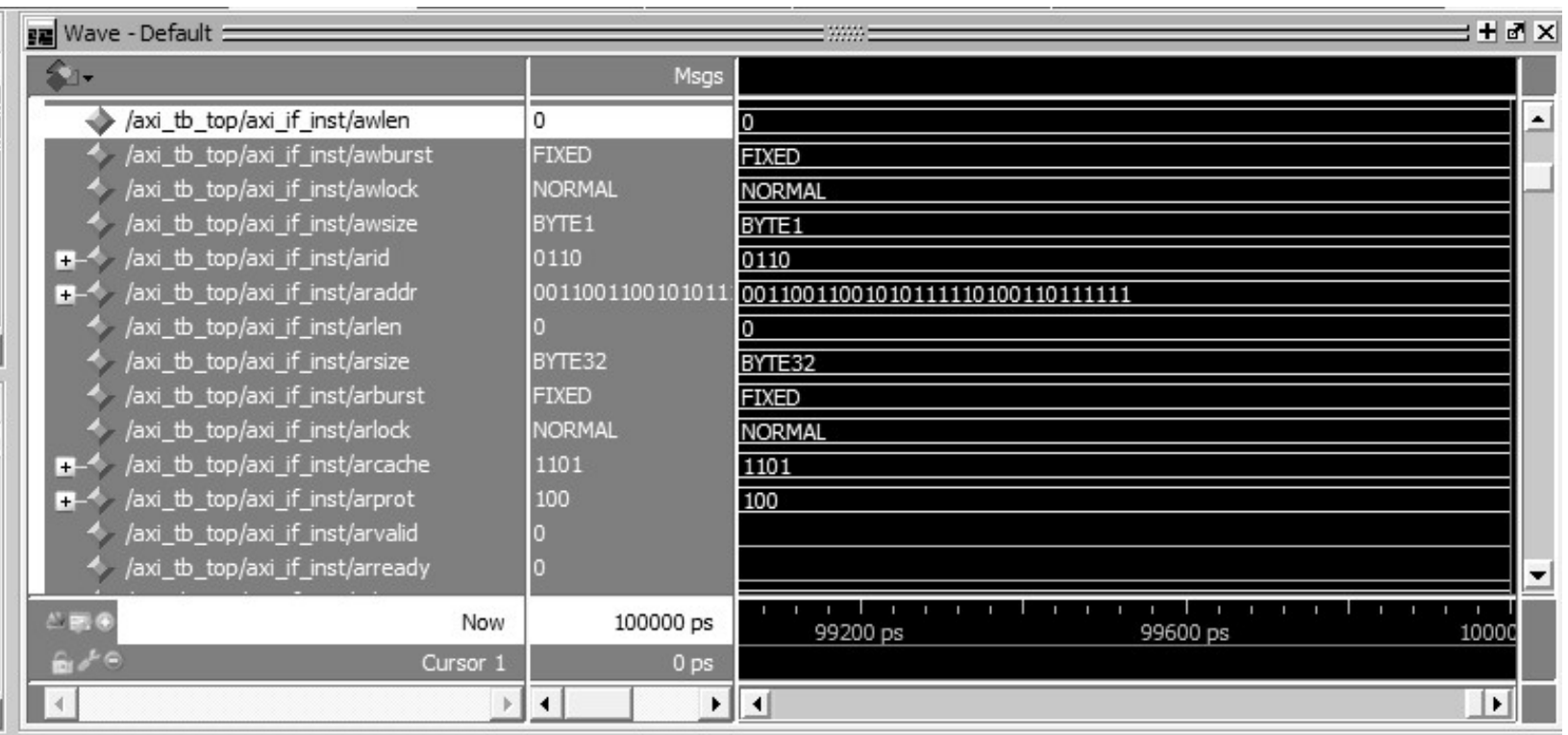

Figure 4: AXI Basic Transaction

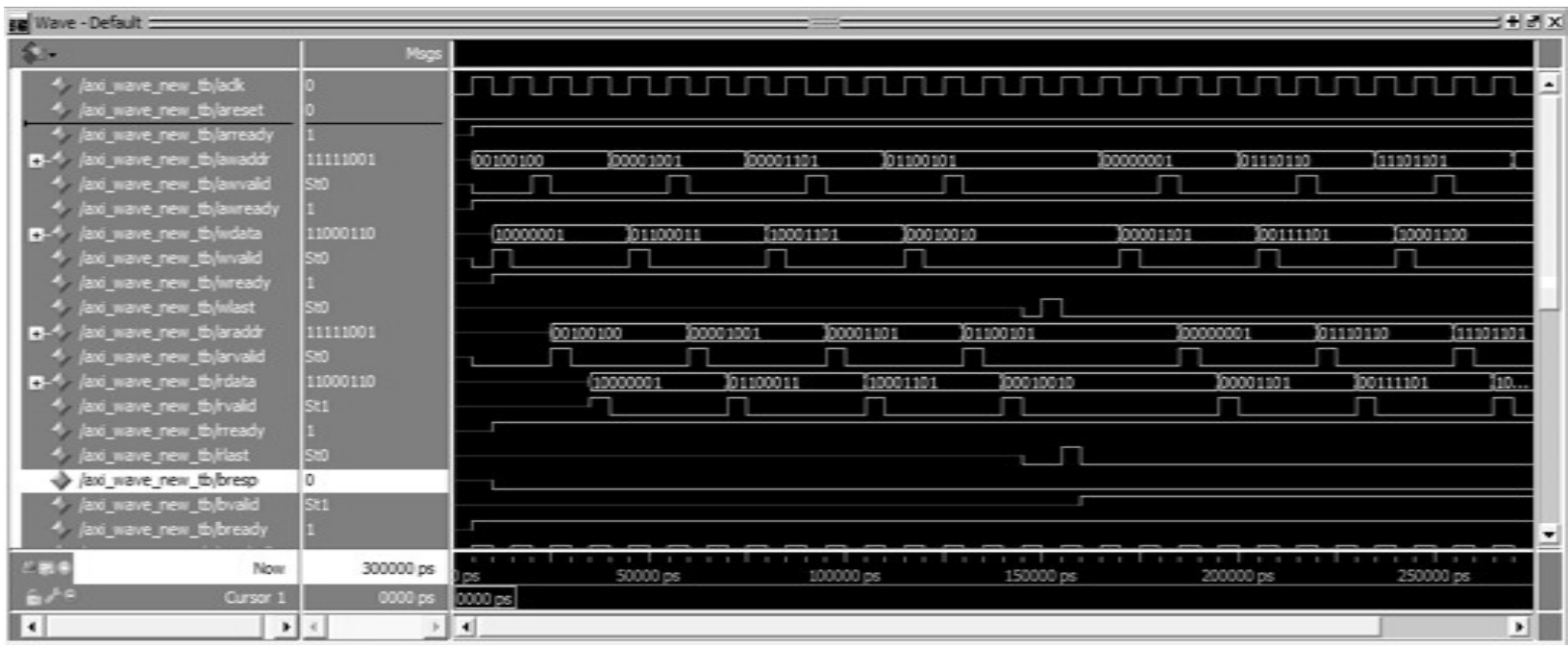

Figure 5: FIXED Burst-type Transaction

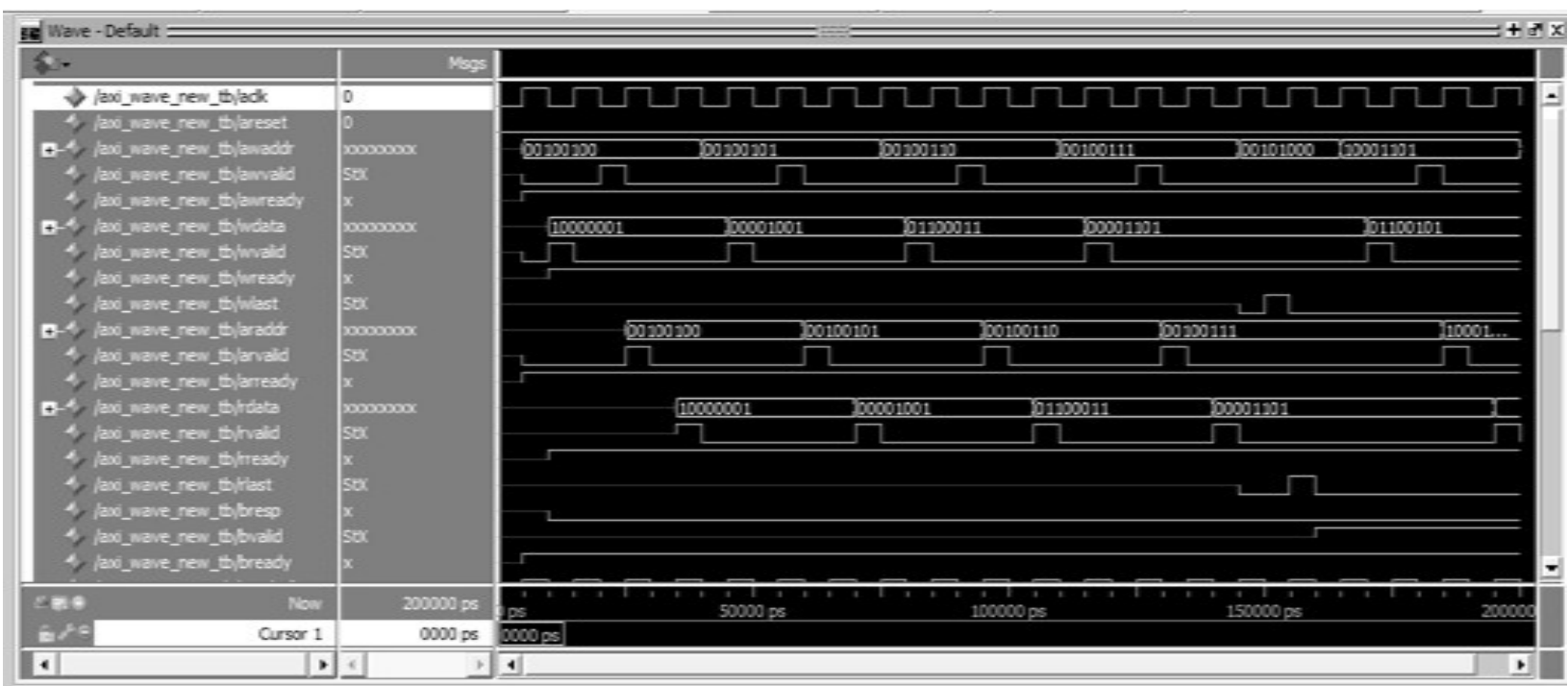

Figure 6: INCR Burst-type Transaction 


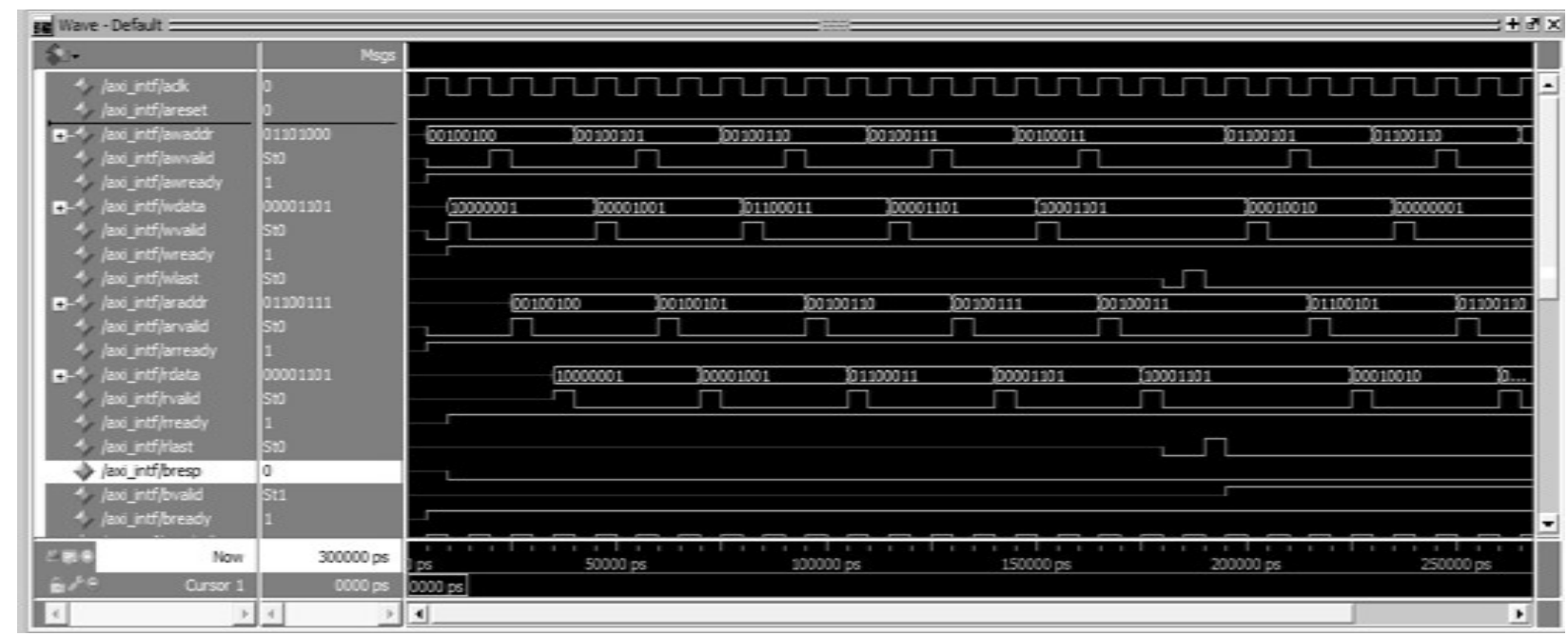

Figure 8: WRAP-4 Burst-type Transaction

\section{SYSTEM VERILOG VERIFICATION REPORT}

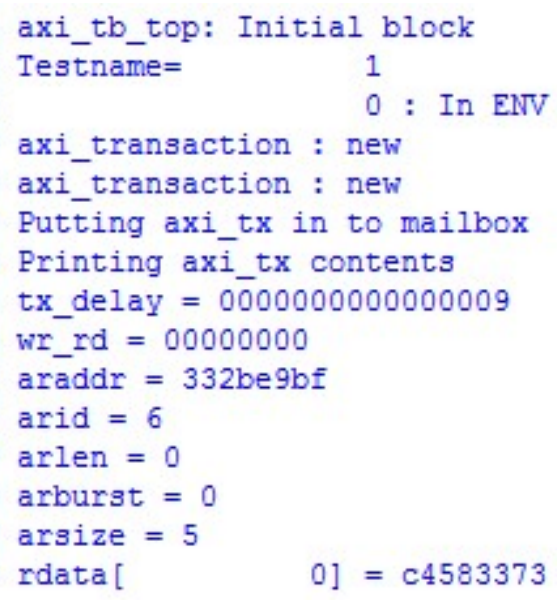

\section{RESULTS}

The results of verification components such as Interface, Driver, Generator, Transaction, Scoreboard, Assertions and Monitor are developed using System Verilog. According to the designed verification environment, the test cases are verified for various burst types implementing AXI Protocol interconnection between a master and a slave. The Test Cases are written in the Generator block by assigning testcase number. The Driver drives the generated packets to the DUV. Various burst-type transactions have been performed in DUV. The DUV output values are compared with the output values from the Golden Model in the checker block.. If the DUV output value matches the Golden Model output value then we conclude that the verification of the design is completed successfully. By using the simulator tool Questa various test cases are generated and simulated which resulted in generation of waveforms shown in Figures 4 to 8.

\section{CONCLUSION}

Verification of AXI Bus Protocol for Single Master-Single Slave implementing FIXED, INCR and WRAP has been achieved by developing the Verification IP using System
Verilog. Test has been passed for various burst size and burst length has been used for:-

- INCR: single address followed by data in sequence has been passed unlike FIXED where address and data was transferred in pipeline.

- WRAP: when boundary was reached data was wrapped to one lower address unlike INCR and FIXED.
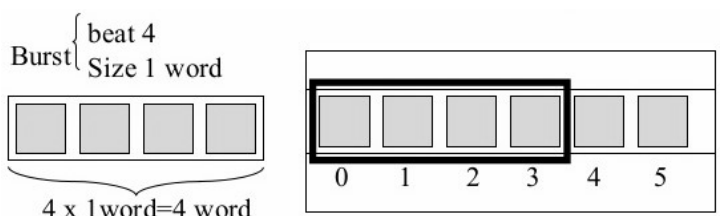

Figure 9: Burst-Size and Burst Length implemented

Verilog", International Journal \& Magazine of Engineering, Technology, Management and Research, Volume No: 2 (2015), Issue No: 7 (July), Page 612, ISSN No: 2348-4845

[5] Mahesh Pai N, H Sudha and Lakshmikantha HN, "Design and Implementation of AMBA based AXI 4 Slave Interface", International Journal of Innovative Research in Science, Engineering and Technology, Vol. 4, Issue 6, June 2015, ISSN(Online): 2319-8753, Page 4253 
\# axi_transaction : new

\# Putting axi_tx in to mailbox

\# Printing axi tx contents

\# tx_delay $=0000000000000003$

\# wr_rd $=00000001$

\# awaddr $=57 \mathrm{c} 23$ eef

\# awid $=7$

\# awlen $=5$

\# awburst $=0$

\# awsize $=1$

\# wdata [ 0$]=$ bee $41 f f 9$

\# wdata $[\quad 1]=845 \mathrm{e} 0 \mathrm{efc}$

\# wdata [ 2] $=$ bdc81f $4 \mathrm{~b}$

\# wdata [ 3] $=85420 \mathrm{e} 5 \mathrm{f}$

\# wdata [ 4] $=8 \operatorname{acclf} 4 \mathrm{f}$

\# wdata [ 5] $=9226087 \mathrm{~d}$

\# wstrb $[0]=2$

\# wstrb [ 1$]=0$

\# watrb $[2]=e$

\# wstrb $[3]=d$

\# wstrb [ 4$]=5$

\# wstrb[ 5$]=\mathrm{b}$

\begin{tabular}{|c|c|c|c|c|}
\hline $\begin{array}{c}\text { Burst- } \\
\text { Type }\end{array}$ & $\begin{array}{c}\text { Burst- } \\
\text { Length }\end{array}$ & $\begin{array}{c}\text { Burst- } \\
\text { Size }\end{array}$ & $\begin{array}{c}\text { Start } \\
\text { Address }\end{array}$ & $\begin{array}{c}\text { End } \\
\text { Address }\end{array}$ \\
\hline FIXED & 4 & 1-Byte & 0x00 & - \\
\hline INCR & 4 & 1-Byte & 0x02 & 0x05 \\
\hline WRAP & 4 & 1-byte & 0x02 & 0x01 \\
\hline
\end{tabular}

An analysis of the verification plan has been made according to the working of AXI protocol for single master and single slave for various burst-type transactions. The study of a successful verification environment resulted in a high coverage verification.

\section{ACKNOWLEDGEMENT}

We the authors of this paper would like to acknowledge with thanks to Mr. Chusen Duari, Assistant Professor, Department of Electronics and Communication Engineering, Manipal University Jaipur for constant encouragement and support for enabling us to submit this paper.

\section{REFERENCES}

Journal / Conference Papers

[1] Xu Chen, Zheng Xie, and Xin-An Wang, "Development of Verification Environment for AXI Bus Using SystemVerilog" (IEEE) International Journal of Electronics and Electrical Engineering Vol. 1, No. 2, June 2013

[2] Xiongfei Liao, Jun Zhou, Liu, X. "Exploring AMBA AXI on-Chip interconnection for SoCs", 3D Systems Integration Conference (3DIC), January 2011 IEEE International, Pages $1-4$

[3] K. Han, Z. Deng, and Q. Shu, "Verification of AMBA bus model using systemverilog," in Proc. 8th International Conference on Electronic Measurement and Instruments, 2007, pp. 1-776-1-780.

[4] Sundararajan PH and S.Balaji, "Verification of AXI Bus Protocol using System

Web:

[1] ARM, "AMBA Specification Overview", http://www.arm.com.
[2] ARM, "AMBA Specification (Rev 2.0)", http://www.arm.com

[3] ARM, "AMBA AXI Protocol Specification", http://www.arm.com

[4] System Verilog and UVM, "System Verilog \& UVM", https://www.doulos.com

\section{BIOGRAPHIES}

- Harsha Garua, M.Tech Digital Electronics and Communication, School of Electrical, Electronics \& Communication Engineering, Manipal University Jaipur.

- Keshav Sharma, M.Tech Microelectronics, School of Electrical, Electronics \& Communication Engineering, Manipal University Jaipur.

- Mr. Chusen Duari, Assistant Professor, Department of Electronics \& Communication Engineering, Manipal University Jaipur Appendix

AMBA - Advanced Microcontroller Based Architecture.

AXI- Advanced Extensible Interface

AHB - Advanced High performance Bus.

APB - Advanced Peripheral Bus.

SOC - System On Chip.

IP - Intellectual Property

DUV- Design Under Verification

INCR- Increment burst

WRAP- Wrapping burst

FIFO- First-In-First-Out 